\title{
518.
}

\section{SUR LA CONDITION POUR QU'UNE FAMILLE DE SURFACES DONNÉES PUISSE FAIRE PARTIE D'UN SYSTÈME ORTHOGONAL.}

[From the Comptes Rendus de l'Académie des Sciences de Paris, tom. Lxxv. (JuilletDécembre, 1872), pp. 177-185, 246-250, 324-330, 381-385, 1800-1803.]

$$
\text { [Pp. 177-185.] }
$$

1. SoIt $\rho=f(x, y, z)$ l'équation d'une famille de surfaces qui fait partie d'un système orthogonal. On sait que $\rho$ satisfait à une équation à différences partielles du troisième ordre, et en suivant la route tracée par M. Levy, dans son excellent "Mémoire sur les coordonnées curvilignes orthogonales" (Journal de l'École Polytechnique, t. xxvi., pp. 157-200, 1870), je suis parvenu à trouver cette équation.

2. Je remarque que le théorème fondamental de $M$. Levy est, en effet, assez évident. Considérons une surface de la famille $\rho$ : soit $P$ un point quelconque de cette surface, et $P T, P T_{1}, P T_{2}$ la normale et les tangentes aux deux courbes de courbure par le point $P$. Passons, suivant la normale au point $P^{\prime}$ de la surface consécutive $\rho+d \rho$, et soient $P^{\prime} T^{\prime}, P^{\prime} T_{1}^{\prime}, P^{\prime} T_{2}^{\prime}$ la normale et les tangentes aux deux courbes de courbure par le point $P^{\prime}$. Or, si les surfaces $\rho$ forment partie d'un système orthogonal, évidemment $P P^{\prime}$ sera élément d'une courbe de courbure d'une surface $\rho_{1}$ et aussi d'une surface $\rho_{2}$ des deux autres familles du système orthogonal, et $P T_{1}, P^{\prime} T_{1}^{\prime}$ seront les normales à deux points consécutifs de cette courbe de courbure de la surface $\rho_{1}$ : et de même $P T_{2}$ et $P^{\prime} T_{2}^{\prime}$ seront les r.srmales à deux points consécutifs de cette courbe de courbure de la surface $\rho_{2}$. Donc $P T_{1}$ et $P^{\prime} T_{1}^{\prime}$ se rencontrent; et de même $P T_{2}$ et $P^{\prime} T_{2}^{\prime}$ se rencontrent. En se souvenant que $P T_{1}, P T_{2}$ sont perpendiculaires l'une à l'autre, et de même $P^{\prime} T_{1}^{\prime}, P^{\prime} T_{2}^{\prime}$, on voit sans peine que les deux conditions se réduisent à une seule. Réciproquement, si $P T_{1}, P^{\prime} T_{1}^{\prime}$ se rencontrent (ou, ce qui est la même chose, $P T_{2}$ et $\left.P^{\prime} T_{2}^{\prime}\right)$, la famille $\rho$ fera partie d'un système orthogonal; ce qui est le théorème de M. Levy. 
3. Soient $(X, Y, Z)$ les fonctions dérivées de $\rho$ du premier ordre; $(\mathrm{a}, \mathrm{b}, \mathrm{c}, \mathrm{f}, \mathrm{g}, \mathrm{h})$ celles du second ordre; $(a, b, c, f, g, h, i, j, k, l)$ celles du troisième ordre, savoir:

$$
(X, Y, Z)=\left(\partial_{x}, \partial_{y}, \partial_{z}\right) \rho,
$$

$$
(\mathrm{a}, \mathrm{b}, \mathrm{c}, \mathrm{f}, \mathrm{g}, \mathrm{h})=\left(\partial_{x}^{2}, \partial_{y}{ }^{2}, \partial_{z}{ }^{2}, \partial_{y} \partial_{z}, \partial_{z} \partial_{x}, \partial_{x} \partial_{y}\right) \rho,
$$

$(a, b, c, f, g, h, i, j, k, l)=\left(\partial_{x}{ }^{3}, \partial_{y}{ }^{3}, \partial_{z}{ }^{3}, \partial_{y}{ }^{2} \partial_{z}, \partial_{z}{ }^{2} \partial_{x}, \partial_{x}{ }^{2} \partial_{y}, \partial_{y} \partial_{z}{ }^{2}, \partial_{z} \partial_{x}{ }^{2}, \partial_{x} \partial_{y}{ }^{2}, \partial_{x} \partial_{y} \partial_{z}\right) \rho ;$

soient de plus

$$
\begin{array}{ll}
A=2(Z \mathrm{~h}-Y \mathrm{~g}), & F=X(\mathrm{c}-\mathrm{h})+Y \mathrm{~h}-Z \mathrm{~g} \\
B=2(X \mathrm{f}-Z \mathrm{~h}), & G=Y(\mathrm{a}-\mathrm{c})+Z \mathrm{f}-X \mathrm{~h} \\
C=2(Y \mathrm{~g}-X \mathrm{f}), & H=Z(\mathrm{~h}-\mathrm{a})+X \mathrm{~g}-Y \mathrm{f}
\end{array}
$$

valeurs qui satisfont aux équations

$$
A+B+C=0 \text { et }(A, B, C, F, G, H \gamma X, Y, Z)^{2}=0 .
$$

Alors les tangentes $P T_{1}, P T_{2}$ sont données par les équations

$$
\begin{array}{r}
(A, B, C, F, G, H \chi x, y, z)^{2}=0, \\
X x+Y y+Z z=0,
\end{array}
$$

et en partant de ces équations, mais en supposant que pour le point $P$ les valeurs de $X, Y$ soient $X=0, Y=0, M$. Levy obtient comme condition de l'intersection dont il s'agit

$$
\left(\frac{d X}{d x}-\frac{d Y}{d y}\right) \frac{d^{2}}{d x d y} \frac{1}{Z}+\frac{d Y}{d x}\left(\frac{d^{2}}{d y^{2}}-\frac{d^{2}}{d x^{2}}\right) \frac{1}{Z}=0
$$

ou, ce qui est la même chose

$$
2 \mathrm{fg}(\mathrm{a}-\mathrm{b})+2 \mathrm{~h}\left(\mathrm{f}^{2}-\mathrm{g}^{2}\right)-Z[(f-j) \mathrm{h}+l(\mathrm{a}-\mathrm{b})]=0 ;
$$

savoir: cette équation est ce que devient l'équation cherchée du troisième ordre en y écrivant $X=0, Y=0$.

4. Je passe à la recherche de l'équation générale; pour cela $(X, Y, Z)$ dénotant comme auparavant, nous pouvons considérer ces quantités comme les coordonnées (mesurées du point $P$ comme origine) d'un point sur la normale $P T$; soient de même $X_{1}, Y_{1}, Z_{1}$ les coordonnées d'un point sur la tangente $P T_{1}$ et $X_{2}, Y_{2}, Z_{2}$ les coordonnées d'un point sur la tangente $P T_{2}$. Il s'agit seulement des valeurs relatives de ces coordonnées; et celles de $X_{1}, Y_{1}, Z_{1}$ et $X_{2}, Y_{2}, Z_{2}$ sont les valeurs de $(x, y, z)$, données par les équations

$$
\begin{aligned}
(A, B, C, F, G, H \chi x, y, z)^{2} & =0, \\
X x+Y y+Z z & =0 .
\end{aligned}
$$


Ces équations impliquent $X_{1} X_{2}+Y_{1} Y_{2}+Z_{1} Z_{2}=0$, et en se rappelant une équation déjà mentionnée, on a le système

$$
\begin{aligned}
& (A, \ldots \gamma X, Y, Z)^{2}=0 \\
& \left(A, \ldots \gamma X_{1}, Y_{1}, Z_{1}\right)^{2}=0 \\
& \left(A, \ldots \gamma X_{2}, Y_{2}, Z_{2}\right)^{2}=0 \\
& X_{1} X_{2}+Y_{1} Y_{2}+Z_{1} Z_{2}=0 \\
& X X_{1}+Y Y_{1}+Z Z_{1}=0 \\
& X X_{2}+Y Y_{2}+Z Z_{2}=0
\end{aligned}
$$

L'origine étant quelconque, prenons $(x, y, z)$ pour coordonnées de $P$, et $x+\delta x, y+\delta y ; z+\delta z$ pour coordonnées de $P^{\prime}$; nous avons $\delta x: \delta y: \delta z=X: Y: Z$; et comme il ne s'agit que des valeurs relatives, nous pouvons omettre un facteur infinitésimal commun, et écrire simplement $\delta x, \delta y, \delta z=X, Y, Z$. De même, en supposant qu'une fonction quelconque $u$ de $(x, y, z)$ devient $u+\delta u$, en passant du point $P$ au point $P^{\prime}$, la valeur de $\delta u$ sera $X \frac{d u}{d x}+Y \frac{d u}{d y}+Z \frac{d u}{d z}$, ou, ce qui est la même chose, nous aurons $\delta=X \frac{d}{d x}+Y \frac{d}{d y}+Z \frac{d}{d z}$. Dans tout ce qui suit, $\delta$ aura cette signification.

5. Cela étant, si pour un moment nous prenons $\xi, \eta, \zeta$ pour coordonnées courantes, et $\theta$ pour un paramètre arbitraire, les équations de $P T$ seront

$$
\xi=x+\theta X_{1}, \quad \eta=y+\theta Y_{1}, \quad \zeta=z+\theta Z_{1},
$$

et si cette droite rencontre $P T_{1}$, alors en prenant $\xi, \eta, \zeta$ pour les coordonnées du point d'intersection, nous aurons $0=\delta x+X_{1} \delta \theta+\theta \delta X_{1}, \ldots$ : ou en éliminant $\delta \theta$ et $\theta$,

$$
0=\left|\begin{array}{lll}
\delta x, & X_{1}, & \delta X_{1} \\
\delta y, & Y_{1}, & \delta Y_{1} \\
\delta z, & Z_{1}, & \delta Z_{1}
\end{array}\right|,
$$

ou, ce qui est la même chose,

$$
0=\left|\begin{array}{ccc}
X, & X_{1}, & \delta X_{1} \\
Y, & Y_{1}, & \delta Y_{1} \\
Z, & Z_{1}, & \delta Z_{1}
\end{array}\right|
$$

Mais nous avons $X_{2}: Y_{2}: Z_{2}=Y Z_{1}-Z Y_{1}: Z X_{1}-X Z_{1}: X Y_{1}-Y X_{1}:$ donc cette équation devient $X_{2} \delta X_{1}+Y_{2} \delta Y_{1}+Z_{2} \delta Z_{1}=0$. Or nous avons $\delta\left(X_{1} X_{2}+Y_{1} Y_{2}+Z_{1} Z_{2}\right)=0$; l'équation trouvée peut donc s'écrire sous la forme plus symétrique

$$
X_{2} \delta X_{1}+Y_{2} \delta Y_{1}+Z_{2} \delta Z_{1}-\left(X_{1} \delta X_{2}+Y_{1} \delta Y_{2}+Z_{1} \delta Z_{2}\right)=0,
$$

équation qui exprime la condition pour l'intersection des tangentes $P T_{1}^{\prime}, P^{\prime} T_{1}^{\prime}$ (ou $\left.P T_{2}, P^{\prime} T_{2}^{\prime}\right)$. 
6. Dans la démonstration précédente, je me suis servi du théorème de Dupin; mais il convient de remarquer qu'en partant du système orthogonal, et dénotant par $X, Y, Z ; X_{1}, Y_{1}, Z_{1} ; X_{2}, Y_{2}, Z_{2}$ les dérivées de $\rho, \rho_{1}, \rho_{2}$, respectivement, il serait possible de déduire cette même équation des seules équations

$$
\begin{aligned}
& X X_{1}+Y Y_{1}+Z Z_{1}=0 \\
& X X_{2}+Y Y_{2}+Z Z_{2}=0 \\
& X_{1} X_{2}+Y_{1} Y_{2}+Z_{1} Z_{2}=0
\end{aligned}
$$

En effet, l'équation fut démontrée de cette manière par R. L. Ellis, dans une démonstration du théorème de Dupin, publiée dans l'ouvrage de Gregory (Examples of the processes of the differential and integral calculus; Cambridge, 1841). Les premières deux équations donnent $X: Y: Z=Y_{1} Z_{2}-Y_{2} Z_{1}: Z_{1} X_{2}-Z_{2} X_{1}: X_{1} Y_{2}-X_{2} Y_{1}$; on a donc l'expression

$$
\left(Y_{1} Z_{2}-Y_{2} Z_{1}\right) d x+\left(Z_{1} X_{2}-Z_{2} X_{1}\right) d y+\left(X_{1} Y_{2}-X_{2} Y_{1}\right) d z,
$$

intégrable par un facteur; ce qui donne

$$
\left(Y_{1} Z_{2}-Y_{2} Z_{1}\right)\left\{\frac{d}{d y}\left(X_{1} Y_{2}-X_{2} Y_{1}\right)-\frac{d}{d z}\left(Z_{1} X_{2}-Z_{2} X_{1}\right)\right\} \cdots=0 .
$$

Le terme en \{\} est égal à

$$
\begin{aligned}
\left(X_{2} \frac{d X_{1}}{d x}+Y_{2} \frac{d X_{1}}{d y}+Z_{2} \frac{d X_{1}}{d z}\right)-\left(X_{1} \frac{d X_{2}}{d x}+Y_{1} \frac{d X_{2}}{d y}+Z_{1} \frac{d X_{2}}{d z}\right) \\
-X_{2}\left(\frac{d X_{1}}{d x}+\frac{d Y_{1}}{d y}+\frac{d Z_{1}}{d z}\right)+X_{1}\left(\frac{d X_{2}}{d x}+\frac{d Y_{2}}{d y}+\frac{d Z_{2}}{d z}\right),
\end{aligned}
$$

et la somme qui correspond à la deuxième ligne de cette expression s'évanouit identiquement; la première ligne peut s'écrire sous la forme $\delta_{2} X_{1}-\delta_{1} X_{2}$; donc, en rétablissant $X, Y, Z$ au lieu de $Y_{1} Z_{2}-Y_{2} Z_{1}, \ldots$, la condition devient simplement

$$
X\left(\delta_{2} X_{1}-\delta_{1} X_{2}\right)+Y\left(\delta_{2} Y_{1}-\delta_{1} Y_{2}\right)+Z\left(\delta_{2} Z_{1}-\delta_{1} Z_{2}\right)=0 .
$$

Mais nous avons

$$
\begin{aligned}
& \delta_{2} X_{1}=X_{2} \frac{d X_{1}}{d x}+Y_{2} \frac{d X_{1}}{d y}+Z_{2} \frac{d X_{1}}{d z},=X_{2} \frac{d X_{1}}{d x}+Y_{2} \frac{d Y_{1}}{d x}+Z_{2} \frac{d Z_{1}}{d x}, \\
& \delta_{1} X_{2}=X_{1} \frac{d X_{2}}{d x}+Y_{1} \frac{d X_{2}}{d y}+Z_{1} \frac{d X_{2}}{d z},=X_{1} \frac{d X_{2}}{d x}+Y_{1} \frac{d Y_{2}}{d x}+Z_{1} \frac{d Z_{2}}{d x}
\end{aligned}
$$

et ainsi $\delta_{2} X_{1}+\delta_{1} X_{2}=\frac{d}{d x}\left(X_{1} X_{2}+Y_{1} Y_{2}+Z_{1} Z_{2}\right)=0$; c'est-à-dire $\delta_{1} X_{2}=-\delta_{2} X_{1}$, et de même $\delta_{1} Y_{2}=-\delta_{2} Y_{1}, \delta_{1} Z_{2}=-\delta_{2} Z_{1}$, et l'équation trouvée se réduit à

$$
X \delta_{2} X_{1}+Y \delta_{2} Y_{1}+Z \delta_{2} Z_{1}=0, \quad \text { ou } X \delta_{1} X_{2}+Y \delta_{1} Y_{2}+Z \delta_{1} Z_{2}=0
$$

on a de même

et

$$
X_{1} \delta X_{2}+Y_{1} \delta Y_{2}+Z_{1} \delta Z_{2}=0 \text {, ou } X_{1} \delta_{2} X+Y_{1} \delta_{2} Y+Z_{1} \delta_{2} Z=0 \text {, }
$$$$
X_{2} \delta_{1} X+Y_{2} \delta_{1} Y+Z_{2} \delta_{1} Z=0, \text { ou } X_{2} \delta X_{1}+Y_{2} \delta Y_{1}+Z_{2} \delta Z_{1}=0,
$$ 
et ainsi l'équation dont il s'agit

$$
X_{2} \delta X_{1}+Y_{2} \delta Y_{1}+Z_{2} \delta Z_{1}-\left(X_{1} \delta X_{2}+Y_{1} \delta Y_{2}+Z_{1} \delta Z_{2}\right)=0 .
$$

On ne savait pas auparavant la signification géométrique de cette équation.

7. Dans la question actuelle, partant de cette équation, je rappelle que les valeurs de $X, Y, Z, X_{1}, Y_{1}, Z_{1}$ sont celles de $(x, y, z)$ données par les équations

$$
(A, B, C, F, G, H \curlyvee x, y, z)^{2}=0, \quad X x+Y y+Z z=0 .
$$

En supposant que ces équations donnent

la condition devient

$$
\begin{aligned}
& X_{1}: Y_{1}: Z_{1}=U+U^{\prime}: V+V^{\prime}: W+W^{\prime}, \\
& X_{2}: Y_{2}: Z_{2}=U-U^{\prime}: V-V^{\prime}: W-W^{\prime},
\end{aligned}
$$

$$
U \delta U^{\prime}+V \delta V^{\prime}+W \delta W^{\prime}-\left(U^{\prime} \delta U+V^{\prime} \delta V+W^{\prime} \delta W\right)=0 .
$$

8. Pour effectuer la réduction de cette formule, nous avons besoin de plusieurs formules subsidiaires. J'écris

$$
\left(B C-F^{2}, C A-G^{2}, A B-H^{2}, G H-A F, H F-B G, F G-C H \chi X, Y, Z\right)^{2}
$$

$$
=\left(\mathfrak{A}, \mathfrak{B},(\mathfrak{C}, \mathfrak{F}, \mathfrak{S}, \mathfrak{S} \chi X, Y, Z)^{2}=-\phi\right. \text {, }
$$

et je dénote par (a), (b), (c), (f), (g), (h) les coefficients de $\lambda^{2}, \ldots$ dans la fonction

savoir, j’écris

$$
(A, B, C, F, G, H \gamma \nu Y-\mu Z, \lambda Z-\nu X, \mu X-\lambda Y)^{2},
$$

$$
\begin{aligned}
& \text { (a) }=B Z^{2}+C Y^{2}-2 F^{\prime} Y Z, \\
& \text { (b) }=C X^{2}+A Z^{2}-2 G Z X, \\
& \text { (c) }=A Y^{2}+B X^{2}-2 H X Y, \\
& \text { (f) }=-A Y Z-F X^{2}+G X Y+H X Z, \\
& \text { (g) }=-B Z X+F X Y-G Y^{2}+H Y Z, \\
& \text { (h) }=-C X Y+F Y Z+G Y Z-H Z^{2},
\end{aligned}
$$

où je remarque qu'en vertu des valeurs de $A, \ldots$ nous avons

$$
\text { (a) }+(b)+(c)=0 \text {. }
$$

Cela étant, nous avons les identités

$$
\begin{aligned}
& {[(\mathrm{a}),(\mathrm{h}),(\mathrm{g})](X, Y, Z)=0,} \\
& {[(\mathrm{~h}),(\mathrm{b}),(\mathrm{f})](X, Y, Z)=0,} \\
& {[(\mathrm{~g}),(\mathrm{f}),(\mathrm{c})](X, Y, Z)=0,}
\end{aligned}
$$

$\left[(b)(c)-(f)^{2},(c)(a)-(g)^{2},(a)(b)-(h)^{2},(g)(h)-(a)(f),(h)(f)-(b)(g),(f)(g)-(c)(h)\right]$

C. VIII.

$$
=-\left(X^{2}, Y^{2}, Z^{2}, Y Z, Z X, X Y\right) \phi \text {, }
$$


savoir, (b) (c) $-(\mathrm{f})^{2}=-X^{2} \phi, \ldots$ De plus

$$
\begin{aligned}
& (A, H, G)[(\mathrm{a}),(\mathrm{h}),(\mathrm{g})]=-X(\mathfrak{H} X+\mathfrak{S} Y+\mathfrak{B} Z)-\phi, \\
& (H, B, F)[(\mathrm{a}),(\mathrm{h}),(\mathrm{g})]=-Y(\mathfrak{H} X+\mathfrak{S} Y+\mathfrak{B S} Z), \\
& (G, F, C)[(\mathrm{a}),(\mathrm{h}),(\mathrm{g})]=-Z(\mathfrak{A} X+\mathfrak{S} Y+\mathfrak{S} Z), \\
& (A, H, G)[(\mathrm{h}),(\mathrm{b}),(\mathrm{f})]=-X(\mathfrak{S} X+\mathfrak{B} Y+\mathfrak{F} Z), \\
& (H, B, F)[(\mathrm{h}),(\mathrm{b}),(\mathrm{f})]=-Y(\mathfrak{S} X+\mathfrak{B} Y+\mathfrak{F} Z)-\phi, \\
& (G, F, C)[(\mathrm{h}),(\mathrm{b}),(\mathrm{f})]=-Z(\mathfrak{S} X+\mathfrak{B} Y+\mathfrak{F} Z), \\
& (A, H, G)[(\mathrm{g}),(\mathrm{f}),(\mathrm{c})]=-X(\mathfrak{S} X+\mathfrak{F} Y+(\mathfrak{S} Z), \\
& (H, B, F)[(\mathrm{g}),(\mathrm{f}),(\mathrm{c})]=-Y(\mathfrak{S} X+\mathfrak{F} Y+(\mathfrak{E} Z), \\
& (G, F, C)[(\mathrm{g}),(\mathrm{f}),(\mathrm{c})]=-Z(\mathfrak{S} X+\mathfrak{F} Y+(\mathfrak{C} Z)-\phi ;
\end{aligned}
$$

aussi

$$
A(\mathrm{a})+B(\mathrm{~b})+C(\mathrm{c})+2 F(\mathrm{f})+2 G(\mathrm{~g})+2 H(\mathrm{~h})+2 \phi=0 \text {. }
$$

Multipliant cette dernière équation par l'un quelconque des coefficients $(a), \ldots$, et réduisant, on obtient six équations; mais je forme seulement celle qui se dérive de (g), savoir, nous avons

$$
\text { (g) } \begin{aligned}
{\left[A(\mathrm{a})+B(\mathrm{~b})+C(\mathrm{c})+2 F^{\prime}(\mathrm{f})\right.} & +2 G(\mathrm{~g})+2 H(\mathrm{~h})] \\
& +2 \phi\left(-B Z X+F^{\prime} X Y-G Y^{2}+H Y Z\right)=0 .
\end{aligned}
$$

Ici la seconde ligne est égale à

$$
2 B[(\mathrm{f})(\mathrm{h})-(\mathrm{b})(\mathrm{g})]-2 F^{[}[(\mathrm{f})(\mathrm{g})-(\mathrm{c})(\mathrm{h})]+2 G\left[(\mathrm{c})(\mathrm{a})-(\mathrm{g})^{2}\right]-2 H[(\mathrm{~g})(\mathrm{h})-(\mathrm{a})(\mathrm{f})],
$$

et l'équation est

$$
A(\mathrm{a})(\mathrm{g})+B[2(\mathrm{~h})(\mathrm{f})-(\mathrm{b})(\mathrm{g})]+C(\mathrm{c})(\mathrm{g})+2 F(\mathrm{c})(\mathrm{h})+2 G(\mathrm{c})(\mathrm{a})+2 H(\mathrm{a})(\mathrm{f})=0 .
$$

Des équations $(\mathrm{g})(\mathrm{h})-(\mathrm{a})(\mathrm{f})=-Y Z \phi$, (h) (f) $-(\mathrm{b})(\mathrm{g})=-Z X \phi$, multipliant par $-X,-Y$ et ajoutant, nous obtenons $-(\mathrm{h})[(\mathrm{g}) X+(\mathrm{f}) Y]+(\mathrm{a})(\mathrm{f}) X+(\mathrm{b})(\mathrm{g}) Y=2 X Y Z$, c'est-à-dire

$$
\text { (a) (f) } X+\text { (b) (g) } Y+\text { (c) (f) } Z=2 X Y Z \text {. }
$$

9. Je reviens à la question principale. A moins de se servir de quantités arbitraires qui rendraient les formules plus complexes, il n'y a pas d'expression symétrique pour les valeurs de $X_{1}: Y_{1}: Z_{1}$ et $X_{2}: Y_{2}: Z_{2}$ : et ainsi j'écris

$$
\begin{aligned}
& X_{1}: Y_{1}: Z_{1}=(\mathrm{a}):(\mathrm{h})+Z \sqrt{\phi}:(\mathrm{g})-Y \sqrt{\phi}, \\
& X_{2}: Y_{2}: Z_{2}=(\mathrm{a}):(\mathrm{h})-Z \sqrt{\phi}:(\mathrm{g})+Y \sqrt{\phi},
\end{aligned}
$$

et la condition devient

$$
[(\mathrm{h}) \delta Z-Z \delta(\mathrm{h})-(\mathrm{g}) \delta Y+Y \delta(\mathrm{g})] \sqrt{\phi}+[(\mathrm{h}) Z-(\mathrm{g}) Y] \delta \sqrt{\phi}=0,
$$

ou, puisque $\delta \sqrt{\phi}=\frac{1}{2 \sqrt{\phi}} \delta \phi$, ceci est

$$
2[(\mathrm{~h}) \delta Z-Z \delta(\mathrm{h})-(\mathrm{g}) \delta Y+Y \delta(\mathrm{g})] \phi+[(\mathrm{h}) Z-(\mathrm{g}) Y] \delta \phi=0,
$$

équation qui contient, comme nous le verrons, le facteur (a); et, en omettant ce facteur, l'équation deviendra symétrique. 
J'écris

$$
\delta(\mathrm{g})=\Delta(\mathrm{g})+\delta^{\prime}(\mathrm{g}), \quad \delta(\mathrm{h})=\Delta(\mathrm{h})+\delta^{\prime}(\mathrm{h}), \quad \delta \phi=\Delta \phi+\delta^{\prime} \phi,
$$

en dénotant par $\Delta$ les parties qui dépendent de $\delta X, \delta Y, \delta Z$, et par $\delta^{\prime}$ celles qui dépendent de $\delta A, \ldots$. La fonction à droite est ainsi la somme des deux parties

$$
\begin{aligned}
& \Omega_{1}=2[(\mathrm{~h}) \delta Z-Z \Delta(\mathrm{h})-(\mathrm{g}) \delta Y+Y \Delta(\mathrm{g})] \phi+[(\mathrm{h}) Z-(\mathrm{g}) Y] \Delta \phi, \\
& \Omega_{2}=2\left[\quad-Z \delta^{\prime} \cdot(\mathrm{h}) \quad+Y \delta^{\prime}(\mathrm{g})\right] \phi+[(\mathrm{h}) Z-(\mathrm{g}) Y] \delta^{\prime} \phi,
\end{aligned}
$$

où cette seconde partie $\Omega_{2}$ est la seule qui contient les dérivées de $\rho$ du troisième ordre.

10. Je réduis l'expression de $\Omega_{1}$. Nous avons

$$
\begin{aligned}
& \Delta(\mathrm{h})=(-C Y+F Z) \delta X+(-C X+G Z \quad) \delta Y+(F X+G Y-2 H Z) \delta Z, \\
& \Delta(\mathrm{g})=(-B Z+F Y) \delta X+(F X-2 G Y+H Z) \delta Y+(-B X+H Y \quad) \delta Z,
\end{aligned}
$$

et de là

$$
\begin{gathered}
\frac{1}{2} \Omega_{1}=\phi\left\{\left[(C-B) Y Z+F\left(Y^{2}-Z^{2}\right)\right] \delta X+\left[-A X Z+G\left(Y^{2}+Z^{2}\right)\right] \delta Y+\left[A X Z+H\left(Y^{2}+Z^{2}\right)\right] \delta Z\right\} \\
+\frac{1}{2}[(\mathrm{~h}) Z-(\mathrm{g}) Y] \Delta \phi,
\end{gathered}
$$

où la dernière ligne est égale à

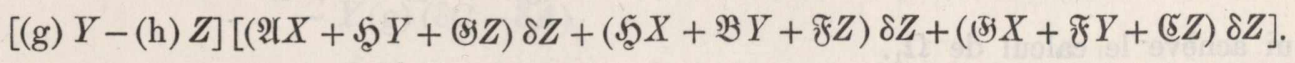

Ici le coefficient de $\delta X$ est égal à

$$
\begin{aligned}
\left.(C-B)[(\mathrm{a})(\mathrm{f})-(\mathrm{g})(\mathrm{h})]+F(\mathrm{~g})^{2}-(\mathrm{a})(\mathrm{c})-(\mathrm{h})^{2}+(\mathrm{a})(\mathrm{b})\right] \\
+[(\mathrm{g}) Y-(\mathrm{h}) Z](\mathfrak{A} X+\mathfrak{S} Y+(\mathrm{s} Z),
\end{aligned}
$$

où la seconde ligne est égale à

$$
-(\mathrm{g})(H, B, F)[(\mathrm{a}),(\mathrm{b}),(\mathrm{g})]+(\mathrm{h})(G, F, C)[(\mathrm{a}),(\mathrm{h}),(\mathrm{g})],
$$

et ainsi l'expression entière se réduit à

$$
\text { (a) }\{-(B-C) \Theta+F[(\mathrm{~b})-(\mathrm{c})]-H(\mathrm{~g})+G(\mathrm{~h})\}
$$

c'est-à-dire le coefficient de $\delta X$ contient le facteur (a).

Le coefficient de $\delta Y$ est

$$
\left[-A X Z-F\left(Y^{2}+Z^{2}\right)\right] \phi+[(\mathrm{g}) Y-(\mathrm{h}) Z](\mathfrak{g} X+\mathfrak{B} Y+\mathfrak{F} Z),
$$

où la seconde partie est

$$
\text { (g) }\left\{-\phi-\left(H, B, F^{\prime}\right)[(\mathrm{h}),(\mathrm{b}),(\mathrm{f})]\right\}+(\mathrm{h})(G, F, C)[(\mathrm{h}),(\mathrm{b}),(\mathrm{f})] \text {; }
$$

on a donc les termes

c'est-à-dire

$$
-\phi\left[(\mathrm{g})+A Z X+G\left(Y^{2}+Z^{2}\right)\right]
$$

$$
-\phi\left[(A-B) Z X+F X Y+G Z^{2}+H Y Z\right] \text {, }
$$


et l'expression entière est ainsi égale à

c'est-à-dire à

$$
\begin{aligned}
(A-B) & {[(\mathrm{h})(\mathrm{f})-(\mathrm{b})(\mathrm{g})]+F[(\mathrm{f})(\mathrm{g})-(\mathrm{c})(\mathrm{h})]+G\left[(\mathrm{a})(\mathrm{b})-(\mathrm{h})^{2}\right] } \\
& +H[(\mathrm{~g})(\mathrm{h})-(\mathrm{a})(\mathrm{f})]-(\mathrm{g})\left(H, B, F^{\prime}\right)[(\mathrm{h}),(\mathrm{b}),(\mathrm{f})] \\
& +(\mathrm{h})(G, F, C)[(\mathrm{h}),(\mathrm{b}),(\mathrm{f})]
\end{aligned}
$$

$$
A[(\mathrm{~h})(\mathrm{f})]-(\mathrm{b})(\mathrm{g})-B(\mathrm{~h})(\mathrm{f})+C(\mathrm{~h})(\mathrm{f})+F^{\prime}[(\mathrm{b})-(\mathrm{c})](\mathrm{h})+G(\mathrm{a})(\mathrm{b})-H(\mathrm{a})(\mathrm{f}),
$$

ou enfin à

$$
A-(\mathrm{b})(\mathrm{g})+B-2(\mathrm{~h})(\mathrm{f})+F[(\mathrm{~b})(\mathrm{c})](\mathrm{h})+G(\mathrm{a})(\mathrm{b})+H-(\mathrm{a})(\mathrm{f}) .
$$

J'ajoute la quantité nulle

$$
\begin{aligned}
A(\mathrm{a})(\mathrm{g})+B[2(\mathrm{~h})(\mathrm{f})-(\mathrm{b})(\mathrm{g})] & +C(\mathrm{c})(\mathrm{g}) \\
+F^{2}(\mathrm{c})(\mathrm{h})+G 2(\mathrm{c})(\mathrm{a}) & +H 2(\mathrm{a})(\mathrm{f})
\end{aligned}
$$

et, en réduisant au moyen de $(a)+(b)+(c)=0$ et $A+B+C=0$, le coefficient de $\delta Y$ devient

$$
=(\mathrm{a})\{-(C-A)(\mathrm{g})+H(\mathrm{f})+G[(\mathrm{c})-(\mathrm{a})]-F(\mathrm{~h})\} \text {, }
$$

et, de même, le coefficient de $\delta \boldsymbol{Z}$ est

$$
=(\mathrm{a})\{-(A-B)(\mathrm{h})-G(\mathrm{f})+F(\mathrm{~g})+H[(\mathrm{a})-(\mathrm{b})]\},
$$

ce qui achève le calcul de $\Omega_{1}$.

[Pp. 246-250.]

11. Pour trouver $\Omega_{2}$, nous avons

$$
\begin{aligned}
& \delta^{\prime}(\mathrm{g})=-Z X \delta B+Y X \delta F^{\prime}-Y^{2} \delta G+Y Z \delta H \\
& \delta^{\prime}(\mathrm{h})=-X Y \delta C+X Z \delta F^{\prime}+Y Z \delta G-Z^{2} \delta H \\
& \delta^{\prime} \phi=-\left[(\mathrm{a}) \delta A+\text { (b) } \delta B-(\mathrm{c}) \delta C+2(\mathrm{f}) \delta F^{\prime}+2(\mathrm{~g}) \delta G+2(\mathrm{~h}) \delta H\right]
\end{aligned}
$$

et de là

$$
\begin{aligned}
\Omega_{2}=2 \phi[ & \left.-X Y Z(\delta B-\delta C)+X\left(Y^{2}-Z^{2}\right) \delta F-Y\left(Y^{2}+Z^{2}\right) \delta G+Z\left(Y^{2}+Z^{2}\right) \delta H\right] \\
& +[(\mathrm{g}) Y-(\mathrm{h}) Z][(\mathrm{a}) \delta A+(\mathrm{b}) \delta B+(\mathrm{c}) \delta C+2(\mathrm{f}) \delta F+2(\mathrm{~g}) \delta G+2(\mathrm{~h}) \delta H],
\end{aligned}
$$

ce qui se réduit tout de suite à

$$
\begin{aligned}
& -[X(\mathrm{a})(\mathrm{f})+Y(\mathrm{~b})(\mathrm{g})+Z(\mathrm{c})(\mathrm{h})](\delta B-\delta C) \\
& +[(\mathrm{g}) Y-(\mathrm{h}) Z][(\mathrm{a}) \delta A+(\mathrm{b}) \delta B+(\mathrm{c}) \delta C] \\
& +2[Y(\mathrm{c})(\mathrm{h})-Z(\mathrm{~b})(\mathrm{g})] \delta F \\
& +2(\mathrm{a})[Y(\mathrm{c})-Z(\mathrm{f}) \quad] \delta G \\
& +2(\mathrm{~g})[Y(\mathrm{f})-Z(\mathrm{~b}) \quad] \delta H .
\end{aligned}
$$

Les premières deux lignes se réduisent facilement à

$$
(a)[-X(f)+Z(h)](\delta B-\delta C)+(a)[(g) Y-(h) Z](\delta A-\delta C),
$$


et la troisième ligne à $2(\mathrm{a})[Z(\mathrm{~g})-X(\mathrm{c})] \delta F$. Donc l'expression entière contient le facteur (a), et nous aurons

$$
\begin{aligned}
\Omega_{2}:(\mathrm{a})= & -[X(\mathrm{f})+Z(\mathrm{~h})](\delta B-\delta C) \\
& +[Y(\mathrm{~g})-Z(\mathrm{~h})](\delta A-\delta C) \\
& +2[Z(\mathrm{~g})-X(\mathrm{c})] \delta F \\
& +2[Y(\mathrm{c})-Z(\mathrm{f})] \delta G \\
& +2[Y(\mathrm{f})-Z(\mathrm{~b})] \delta H,
\end{aligned}
$$

expression qui se réduit sans peine à la forme symétrique sous laquelle je la présente dans l'équation finale.

12. Cette équation est $\Omega_{1}+\Omega_{2}=0$; savoir, en omettant le facteur (a), nous avons

$$
\begin{aligned}
2 & {[\{F[(\mathrm{~b})-(\mathrm{c})]-(B-C)(\mathrm{f})-H(\mathrm{~g})+G(\mathrm{~h}) \quad\} \delta X} \\
& +\{G[(\mathrm{c})-(\mathrm{a})]+H(\mathrm{f})-(C-A)(\mathrm{g})-F(\mathrm{~h})\} \delta Y \\
& +\{H[(\mathrm{a})-(\mathrm{b})]-G(\mathrm{~h})+F(\mathrm{~g})-(A-B)(\mathrm{h})\} \delta Z] \\
& -X(\mathrm{f})(\delta B-\delta C) \\
& -Y(\mathrm{~g})(\delta C-\delta A) \\
& -Z(\mathrm{~h})(\delta A-\delta B) \\
& +\{X[(\mathrm{~b})-(\mathrm{c})]-Y(\mathrm{~h})+Z(\mathrm{~g})\} \delta F \\
& +\{X(\mathrm{~h})+Y[(\mathrm{c})-(\mathrm{a})]-Z(\mathrm{f})\} \delta G \\
& +\{-X(\mathrm{~g})+Y(\mathrm{f})+Z[(\mathrm{a})-(\mathrm{b})]\} \delta H=0 .
\end{aligned}
$$

On se rappelle que $\delta$ signifie

$$
X \frac{d}{d x}+Y \frac{d}{d y}+Z \frac{d}{d z}
$$

1.3. Pour déduire de là le résultat de M. Levy, j'écris d'abord $X=0, Y=0$; nous avons alors

$$
[(\mathrm{a}),(\mathrm{b}),(\mathrm{c}),(\mathrm{f}),(\mathrm{g}),(\mathrm{h})]=\left(B Z^{2}, A Z^{2}, 0,0,0,-H Z^{2}\right),
$$

et l'équation devient

$$
2[(A F-G H) \delta X+(-B G+F H) \delta Y]+H Z(\delta A-\delta B)-Z(A-B) \delta \Pi=0 ;
$$

mais ici

$$
(A, B, C, F, G, H)=[2 Z \mathrm{~h},-2 Z \mathrm{~h}, 2 \mathrm{~h}, 0,-Z \mathrm{~g}, Z \mathrm{f},-Z(\mathrm{a}-\mathrm{b})],
$$

et l'équation devient

$$
2\{[\mathrm{f}(\mathrm{a}-\mathrm{b})-2 \mathrm{gh}] \delta X+[\mathrm{g}(\mathrm{a}-\mathrm{b})+2 \mathrm{fh}] \delta Y\}-(\mathrm{a}-\mathrm{b})(\delta A-\delta B)-4 \mathrm{~h} \delta H=0 .
$$


Mais nous avons $\delta X=\mathrm{g} Z, \delta Y=\mathrm{f} Z, \delta Z=\mathrm{c} Z$, et, de plus,

$$
\begin{aligned}
& \delta A=2 \delta Z \mathrm{~h}-2 \mathrm{~g} \delta Y=2 l Z^{2}+2(\mathrm{ch}-\mathrm{fg}) Z \\
& \delta B=2 \mathrm{f} \delta X-2 \delta Z \mathrm{~h}=-2 l Z^{2}-2(\mathrm{ch}-\mathrm{fg}) Z, \\
& \delta H=-\delta Z(\mathrm{a}-\mathrm{b})+\mathrm{g} \delta X-\mathrm{f} \delta Y=(f-j) Z^{2}+\left(-\mathrm{ac}+\mathrm{bc}-\mathrm{f}^{2}+\mathrm{g}^{2}\right) Z
\end{aligned}
$$

l'équation est donc

$4 \mathrm{fg}(\mathrm{a}-\mathrm{b})+4\left(\mathrm{f}^{2}-\mathrm{g}^{2}\right) \mathrm{h}-(\mathrm{a}-\mathrm{b})[4 l Z+4(\mathrm{ch}-\mathrm{fg})]-4 \mathrm{~h}\left[-\mathrm{c}(\mathrm{a}-\mathrm{b})-\left(\mathrm{f}^{2}-\mathrm{g}^{2}\right)+(f-j) Z\right]=0$, ou enfin

$$
2 \mathrm{fg}(\mathrm{a}-\mathrm{b})+2 \mathrm{~h}\left(\mathrm{f}^{2}-\mathrm{g}^{2}\right)-Z[(f-j) \mathrm{h}+l(\mathrm{a}-\mathrm{b})]=0,
$$

ce qui s'accorde avec le résultat cité.

14. En changeant la signification de $X, Y, Z$, écrivons $\rho=X+Y+Z$, où $X, Y, Z$ dénotent à présent des fonctions de $x, y, z$ respectivement; en dénotant par $X^{\prime}, Y^{\prime}, Z^{\prime}$ les fonctions dérivées de celles-ci, les fonctions premièrement représentées par $X, Y, Z$ seront $X^{\prime}, Y^{\prime}, Z^{\prime}$. Je cherche, au moyen de l'équation générale, la condition pour que la famille $\rho=X+Y+Z$ puisse faire partie d'un système orthogonal.

Dénotons par $X^{\prime}, X^{\prime \prime}, X^{\prime \prime \prime}$ les dérivées de $X$, et de même celles de $Y$ et $Z$, et écrivons, pour abréger, $\alpha, \beta, \gamma=Y^{\prime \prime}-Z^{\prime \prime}, Z^{\prime \prime}-X^{\prime \prime}, X^{\prime \prime}-Y^{\prime \prime}$, nous avons

$$
(\mathrm{a}, \mathrm{b}, \mathrm{c}, \mathrm{f}, \mathrm{g}, \mathrm{h})=\left(X^{\prime \prime}, Y^{\prime \prime}, Z^{\prime \prime}, 0,0,0\right),
$$

et de là

et, de plus,

$$
(A, B, C, F, G, H)=\left(0,0,0,-\alpha X^{\prime},-\beta Y^{\prime},-\gamma^{\prime}\right),
$$

$$
\begin{aligned}
& {[(\mathrm{a}),(\mathrm{b}),(\mathrm{c}),(\mathrm{f}),(\mathrm{g}),(\mathrm{h})]=\left[2 \alpha X^{\prime} Y^{\prime} Z^{\prime}, 2 \beta X^{\prime} Y^{\prime \prime} Z^{\prime}, 2 \gamma X^{\prime} Y^{\prime} Z^{\prime}\right. \text {, }} \\
& X^{\prime}\left(\alpha X^{\prime 2}-\beta Y^{\prime 2}-\gamma Z^{\prime 2}\right) \text {, } \\
& Y^{\prime}\left(-\alpha X^{\prime 2}+\beta Y^{\prime 2}-\gamma Z^{\prime 2}\right) \text {, } \\
& \left.Z^{\prime}\left(-\alpha X^{\prime 2}-\beta Y^{\prime 2}+\gamma Z^{\prime 2}\right)\right] \text {. }
\end{aligned}
$$

Nous avons aussi

$$
\begin{aligned}
\left(\delta X^{\prime}, \delta Y^{\prime}, \delta Z^{\prime}\right)= & \left(X^{\prime} X^{\prime \prime}, Y^{\prime} Y^{\prime \prime}, Z^{\prime} Z^{\prime \prime}\right) \\
(\delta A, \delta B, \delta C)= & (0,0,0) \\
(\delta F, \delta G, \delta H)= & {\left[X^{\prime}\left(-\alpha X^{\prime \prime}+Z^{\prime} Z^{\prime \prime \prime}-Y^{\prime} Y^{\prime \prime \prime}\right)\right.} \\
& Y^{\prime}\left(-\beta Y^{\prime \prime}+X^{\prime} X^{\prime \prime \prime}-Z^{\prime} Z^{\prime \prime \prime}\right) \\
& \left.Z^{\prime}\left(-\gamma Z^{\prime \prime}+Y^{\prime} Y^{\prime \prime \prime}-X^{\prime} X^{\prime \prime \prime}\right)\right]
\end{aligned}
$$

15. Donc, dans l'équation générale, la première ligne est

$$
\begin{aligned}
2\left[-\alpha X^{\prime} .2 X^{\prime} Y^{\prime} Z^{\prime}(\beta-\gamma)\right. & +\gamma Y^{\prime} Z^{\prime}\left(-\alpha X^{\prime 2}+\beta Y^{\prime 2}-\gamma Z^{\prime 2}\right) \\
& \left.-\beta Y^{\prime} Z^{\prime}\left(-\alpha X^{\prime 2}-\beta Y^{\prime 2}+\gamma Z^{\prime 2}\right)\right] X^{\prime} X^{\prime \prime},
\end{aligned}
$$

c'est-à-dire

$$
\begin{array}{r}
2 X^{\prime} Y^{\prime} Z^{\prime} \cdot X^{\prime \prime}\left[-2 \alpha(\beta-\gamma) X^{\prime 2}+\gamma\left(-\alpha X^{\prime 2}+\beta Y^{\prime 2}-\gamma Z^{\prime 2}\right)\right. \\
\left.-\beta\left(-\alpha X^{\prime 2}-\beta Y^{\prime 2}+\gamma Z^{\prime 2}\right)\right]
\end{array}
$$


ou, ce qui est la même chose,

$$
2 X^{\prime} Y^{\prime} Z^{\prime} \cdot \alpha X^{\prime \prime}\left[(\gamma-\beta) X^{\prime 2}-\beta Y^{\prime 2}+\gamma Z^{\prime 2}\right],
$$

et la somme des premières trois lignes sera aussi $=2 X^{\prime} Y^{\prime} Z^{\prime}$ multiplié par

$$
\begin{aligned}
& \alpha X^{\prime \prime}\left[(\gamma-\beta) X^{\prime 2}-\beta Y^{\prime 2}+\gamma Z^{\prime 2}\right] \\
+ & \beta Y^{\prime \prime}\left[\alpha X^{\prime 2}+(\alpha-\gamma) Y^{\prime 2}-\gamma Z^{\prime 2}\right] \\
+ & \gamma Z^{\prime \prime}\left[-\alpha X^{\prime 2}+\beta Y^{\prime 2}+(\beta-\alpha) Z^{\prime 2}\right],
\end{aligned}
$$

savoir dans ce second facteur le coefficient de $\alpha X^{\prime 2}$ est $X^{\prime \prime}(\gamma-\beta)+\beta Y^{\prime \prime}-\gamma Z^{\prime \prime},=-2 \beta \gamma$, et de même les coefficients de $\beta Y^{\prime 2}$ et $\gamma Z^{\prime 2}$ sont $-2 \gamma \alpha,-2 \alpha \beta$ respectivement, donc le terme entier, ou première partie de l'équation est

$$
4 X^{\prime} Y^{\prime} Z^{\prime}\left(X^{\prime 2}+Y^{\prime 2}+Z^{\prime 2}\right)(-\alpha \beta \gamma)
$$

Les termes en $\delta A, \delta B, \delta C$ s'évanouissent, et il ne reste que les termes en $\delta F, \delta G, \delta H$ qui forment la seconde partie de l'équation. Le premier de ceux-ci est

$$
\begin{aligned}
{\left[X^{\prime} .2(\beta-\gamma) X^{\prime} Y^{\prime} Z^{\prime}\right.} & -Y^{\prime} Z^{\prime}\left(-\alpha X^{\prime 2}-\beta Y^{\prime 2}+\gamma Z^{\prime 2}\right) \\
& \left.+Y^{\prime} Z^{\prime}\left(-\alpha X^{\prime 2}+\beta Y^{\prime 2}-\gamma Z^{\prime 2}\right)\right] \times X^{\prime}\left(-X^{\prime \prime} \alpha+Z^{\prime} Z^{\prime \prime \prime}-Y^{\prime} Y^{\prime \prime \prime}\right)
\end{aligned}
$$

c'est-à-dire

$$
2 X^{\prime} Y^{\prime} Z^{\prime}\left[(\beta-\gamma) X^{\prime 2}+\beta Y^{\prime 2}-\gamma Z^{\prime 2}\right]\left(-X^{\prime \prime} \alpha+Z^{\prime} Z^{\prime \prime \prime}-Y^{\prime} Y^{\prime \prime \prime}\right)
$$

On a donc $2 X^{\prime} Y^{\prime} Z^{\prime}$ multiplié par

$$
\begin{aligned}
& {\left[(\beta-\gamma) X^{\prime 2}+\beta Y^{\prime 2}-\gamma Z^{\prime 2}\right]\left(-X^{\prime \prime} \alpha+Z^{\prime} Z^{\prime \prime \prime}-Y^{\prime} Y^{\prime \prime \prime}\right)} \\
& +\left[-\alpha X^{\prime 2}+(\gamma-\alpha) Y^{\prime 2}+\gamma Z^{\prime 2}\right]\left(-Y^{\prime \prime} \beta+X^{\prime} X^{\prime \prime \prime}-Z^{\prime} Z^{\prime \prime \prime}\right) \\
& +\left[\alpha X^{\prime 2}-\beta Y^{\prime 2}+(\alpha-\beta) Z^{\prime 2}\right]\left(-Z^{\prime \prime} \gamma+Y^{\prime} Y^{\prime \prime \prime}-X^{\prime} X^{\prime \prime \prime}\right)
\end{aligned}
$$

où dans le second facteur nous avons d'abord le terme $-2 \alpha \beta \gamma \times\left(X^{\prime 2}+Y^{\prime 2}+Z^{\prime 2}\right)$ et puis le terme $-2\left(\alpha X^{\prime} X^{\prime \prime \prime}+\beta Y^{\prime} Y^{\prime \prime \prime}+\gamma Z^{\prime} Z^{\prime \prime \prime}\right) \times\left(X^{\prime 2}+Y^{\prime 2}+Z^{\prime 2}\right)$.

La seconde partie est donc

$$
4 X^{\prime} Y^{\prime} Z^{\prime}\left(X^{\prime 2}+Y^{\prime 2}+Z^{\prime 2}\right)\left[-\alpha \beta \gamma-\left(\alpha X^{\prime} X^{\prime \prime \prime}+\beta Y^{\prime} Y^{\prime \prime \prime}+\gamma Z^{\prime} Z^{\prime \prime \prime}\right)\right]
$$

et en réunissiant les deux parties et en omettant le facteur $-4 X^{\prime} Y^{\prime} Z^{\prime} \times\left(X^{\prime 2}+Y^{\prime 2}+Z^{\prime 2}\right)$, l'équation devient

$$
2 \alpha \beta \gamma+\alpha X^{\prime} X^{\prime \prime \prime}+\beta Y^{\prime} Y^{\prime \prime \prime}+\gamma Z^{\prime} Z^{\prime \prime \prime}=0,
$$

savoir :

$2\left(I^{\prime \prime \prime}-Z^{\prime \prime}\right)\left(Z^{\prime \prime}-X^{\prime \prime}\right)\left(X^{\prime \prime}-Y^{\prime \prime}\right)+\left(Y^{\prime \prime}-Z^{\prime \prime}\right) X^{\prime} X^{\prime \prime \prime}+\left(Z^{\prime \prime}-X^{\prime \prime}\right) Y^{\prime} Y^{\prime \prime \prime}+\left(X^{\prime \prime}-Y^{\prime \prime}\right) Z^{\prime} Z^{\prime \prime \prime}=0$,

équation trouvée par M. Bouquet dans sa "Note sur les surfaces orthogonales" (Journal de M. Liouville, t. xI., pp. 446-450, 1846), et reproduite par M. Serret dans son "Mémoire sur les surfaces orthogonales" (Journal de M. Lioniville, t. xiI., pp. 241-254, 1847). 
[Pp. 324-330.]

En considérant une famille orthogonale (savoir: une famille de surfaces qui fait partie d'un système orthogonal), on peut se proposer la question: Étant donnée une surface de la famille, trouver de la manière la plus générale la famille. J'essaye de résoudre cette question en développant les trois coordonnées selon les puissances d'un paramẹtre; et, quoique je n'aie encore calculé que les trois premiers termes des trois développements, les résultats me paraissent assez intéressants pour les soumettre aux géomètres.

On peut, pour la surface donnée, considérer les coordonnées $x, y, z$ d'un point quelconque de la surface comme des fonctions déterminées de deux paramètres $p, q$. $\mathrm{Si}$, de plus, ces paramètres sont tels, que les équations des deux systèmes de courbes de courbure soient $p=$ const., $q=$ const. respectivement, alors (en écrivant pour abréger

$$
\frac{d x}{d p}=x_{1}, \quad \frac{d x}{d q}=x_{2}, \quad \frac{d^{2} x}{d p^{2}}=x_{3}, \quad \frac{d^{2} x}{d p d q}=x_{4}, \quad \frac{d^{2} x}{d q^{2}}=x_{5},
$$

et de même pour $y$ et $z$ ) ces coordonnées $x, y, z$, considérées toujours comme des fonctions de $p, q$, seront telles, que

$$
x_{1} x_{2}+y_{1} y_{2}+z_{1} z_{2}=0, \quad\left|\begin{array}{ccc}
x_{1} & y_{1} & z_{1} \\
x_{2} & y_{2} & z_{2} \\
x_{4} & y_{4} & z_{4}
\end{array}\right|=0 .
$$

J'écris ici et dans la suite $X, Y, Z=y_{1} z_{2}-y_{2} z_{1}, z_{1} x_{2}-z_{2} x_{1}, x_{1} y_{2}-x_{2} y_{1}$. On a donc identiquement

$$
\begin{aligned}
& X x_{1}+Y y_{1}+Z z_{1}=0 \\
& X x_{2}+Y y_{2}+Z z_{2}=0
\end{aligned}
$$

et les deux équations mentionnées sont

$$
\begin{aligned}
& x_{1} x_{2}+y_{1} y_{2}+z_{1} z_{2}=0, \\
& X x_{4}+Y y_{4}+Z z_{4}=0 .
\end{aligned}
$$

Je m'arrête pour remarquer que la dernière équation, dans sa forme originale, peut être remplacée par trois équations de la forme $x_{4}+A x_{1}+B x_{2}=0$, et qu'en ajoutant les trois équations multipliées par $x_{1}, y_{1}, z_{1}$ respectivement, et aussi multipliées par $x_{2}, y_{2}, z_{2}$ respectivement, on obtient les valeurs de $A, B$, exprimées en termes de

$$
E=x_{1}{ }^{2}+y_{1}{ }^{2}+z_{1}{ }^{2} \text { et } G=x_{2}{ }^{2}+y_{2}{ }^{2}+z_{2}{ }^{2}
$$

( $E, G$ de Gauss), et que l'on trouve de là

$$
2 \frac{d^{2} x}{d p d q}-\frac{1}{E} \frac{d E}{d q} \frac{d x}{d p}-\frac{1}{G} \frac{d G}{d p} \frac{d x}{d q}=0,
$$

avec les équations semblables en $y$ et $z$. Ces équations sont, en effet, les équations (10 bis) de Lamé, "Mémoire sur les coordonnées curvilignes" (Liouville, t. v. 1840, p. 322). 
Je suppose que les surfaces de la famille dépendent du paramètre $r$, lequel pour la surface donnée se réduit à $r=0$. Par le point $(p, q)$ de la surface donnée on peut mener une trajectoire orthogonale aux différentes surfaces de la famille; les coordonnées $\xi, \eta, \zeta$ d'un point quelconque sur cette courbe seront des fonctions de $p, q, r$, lesquelles, pour $r=0$, se réduisent à $x, y, z$ respectivement; et j'écris

$$
\begin{aligned}
& \xi=x+a r+d r^{2}+\ldots, \\
& \eta=y+b r+e r^{2}+\ldots, \\
& \zeta=z+c r+f r^{2}+\ldots,
\end{aligned}
$$

où $a, b, c, d, e, f, \ldots$ sont des fonctions inconnues de $p$ et $q$.

Pour exprimer que la courbe coupe orthogonalement les différentes surfaces de la famille, écrivons pour abréger

$$
\begin{array}{ll}
\eta_{1} \zeta_{2}-\eta_{2} \zeta_{1}=X+A r+D r^{2}+\ldots, & X=y_{1} z_{2}-y_{2} z_{1} \\
\zeta_{1} \xi_{2}-\zeta_{2} \xi_{1}=Y+B r+E r^{2}+\ldots, & A=y_{1} c_{2}-y_{2} c_{1}+b_{1} z_{2}-b_{2} z_{1} \\
\xi_{1} \eta_{2}-\xi_{2} \eta_{1}=Z+C r+F r^{2}+\ldots, & \ldots \ldots
\end{array}
$$

(où $\xi_{1}=\frac{d \xi}{d p} \ldots$, comme pour $\left.x, y, z\right)$. La condition cherchée est

$$
\frac{X+A r+D r^{2}+\ldots}{a+2 d r+\ldots}=\frac{Y+B r+E r^{2}+\ldots}{b+2 e r+\ldots}=\frac{Z+C r+F r^{2}+\ldots}{c+2 f r+\ldots},
$$

laquelle doit être satisfaite pour une valeur quelconque de $r$; on a donc

$$
\begin{gathered}
\frac{X}{a}=\frac{Y}{b}=\quad \frac{Z}{c}, \\
\frac{A}{a}-\frac{2 d X}{a^{2}}=\frac{B}{b}-\frac{2 e Y}{b^{2}}=\frac{C}{c}-\frac{2 f Z}{c^{2}} ;
\end{gathered}
$$

savoir, les équations (1) contiennent $(a, b, c)$, les équations (2) contiennent de plus $(d, e, f)$, et ainsi de suite.

Pour qu'il y ait un système orthogonal, il faut et il suffit que l'on ait

$$
\xi_{1} \xi_{2}+\eta_{1} \eta_{2}+\zeta_{1} \zeta_{2}=0
$$

pour toute valeur de $r$; on aura donc

$$
\begin{aligned}
& x_{1} x_{2}+y_{1} y_{2}+z_{1} z_{2}=0, \\
& x_{1} a_{2}+x_{2} a_{1}+y_{1} b_{2}+y_{2} b_{1}+z_{1} c_{2}+z_{2} c_{1}=0, \\
& x_{1} d_{2}+x_{2} d_{1}+y_{1} e_{2}+y_{2} e_{1}+z_{1} f_{2}+z_{2} f_{1}+a_{1} a_{2}+b_{1} b_{2}+c_{1} c_{2}=0,
\end{aligned}
$$

savoir, l'équation [0] est satisfaite d'elle-même; l'équation [1] contient $(a, b, c)$, l'équation [2] contient de plus $(d, e, f)$, et ainsi de suite.

c. VIII. 
Il paraît donc qu'il y a les trois équations (1), [1] pour déterminer $(a, b, c)$; les trois équations (2), [2] pour déterminer $(d, e, f)$, et ainsi de suite. Mais les choses ne se comportent pas ainsi. On satisfait à (1), [1] par des valeurs de $(a, b, c)$ qui contiennent une fonction arbitraire $\lambda$, fonction qui est ensuite déterminée au moyen d'une équation à différences partielles du second ordre, obtenue au moyen des équations (2), [2]; on satisfait alors à (2), [2] par des valeurs de $(d, e, f)$ qui contiennent une fonction arbitraire $\theta$; je présume que cette fonction serait ensuite déterminée au moyen des équations (3), [3], et ainsi de suite; mais je n'ai pas encore fait les calculs ultérieurs.

Par rapport à $\lambda$, en remplaçant cette fonction par $\rho=\lambda \sqrt{X^{2}+Y^{2}+Z^{2}}$, l'équation pour $\rho$ est

$$
2 \frac{d^{2} \rho}{d p d q}-\frac{1}{E} \frac{d E}{d q} \frac{d \rho}{d p}-\frac{1}{G} \frac{d G}{d p} \frac{d \rho}{d q}=0
$$

savoir, c'est la même équation que pour $x, y, z$ : ainsi l'on y satisfait en prenant $\rho$ égal à une fonction linéaire (avec terme constant) quelconque de $x, y, z$.

Pour obtenir ces conclusions, partant des équations (1), [1], les équations (1) donnent

$$
a, b, c=\lambda X, \lambda Y, \lambda Z,
$$

où $\lambda$ est une fonction de $p, q$ : ces valeurs satisfont d'elles-mêmes à l'équation [1]. La vérification se fait sans peine; j'écris pour abréger $x_{1} x_{2}$ pour dénoter $x_{1} x_{2}+y_{1} y_{2}+z_{1} z_{2}$, et ainsi dans les cas semblables: l'équation à vérifier est donc

c'est-à-dire

$$
x_{1}(\lambda X)_{2}+x_{2}(\lambda X)_{1}=0
$$

où nous avons

$$
\lambda\left(x_{1} X_{2}+x_{2} X_{1}\right)+\lambda_{2} x_{1} X+\lambda_{1} x_{2} X=0,
$$

$$
x_{1} X=0, \quad x_{2} X=0 ;
$$

reste à trouver le coefficient $x_{1} X_{2}+x_{2} X_{1}$. Nous avons

et de là

$$
X=y_{1} z_{2}-y_{2} z_{1}
$$

$$
\begin{aligned}
& X_{1}=y_{1} z_{4}-y_{4} z_{1}+y_{3} z_{2}-y_{2} z_{3}, \\
& X_{2}=y_{1} z_{5}-y_{5} z_{1}+y_{4} z_{2}-y_{2} z_{4},
\end{aligned}
$$

et de là, en faisant la somme des trois termes de $x_{1} X_{2}$ et $x_{2} X_{1}$ respectivement, on trouve

$$
x_{1} X_{2}=-\left|\begin{array}{lll}
x_{1}, & y_{1}, & z_{1} \\
x_{2}, & y_{2}, & z_{2} \\
x_{4}, & y_{4}, & z_{4}
\end{array}\right|=x_{2} X_{1}
$$

savoir: $x_{1} X_{2}+x_{2} X_{1}$ est égal à -2 multiplié par ce déterminant, $=-2 X x_{4}$, c'est-à-dire $x_{1} X_{2}+x_{2} X_{1}=0$. Donc la fonction $\lambda$ est jusqu'ici indéterminée. 
Passons aux équations (2), [2]. Substituant dans (2) les valeurs de $(a, b, c)$, ces équations deviennent

$$
\frac{A}{\lambda X}-\frac{2 d}{\lambda^{2} X}=\frac{B}{\lambda Y}-\frac{2 e}{\lambda^{2} Y}=\frac{C}{\lambda Z}-\frac{2 f}{\lambda^{2} Z} .
$$

On y satisfait en écrivant

$$
2 d, 2 e, 2 f=\lambda(\theta X+A), \quad \lambda(\theta Y+B), \quad \lambda(\theta Z+C),
$$

où $\theta$ est fonction de $(p, q)$; en substituant ces valeurs dans l'équation [2], la fonction $\theta$ disparaît d'elle-même; mais on obtient pour $\lambda$ une équation linéaire entre $\lambda, \lambda_{1}, \lambda_{2}$ et $\lambda_{4}$, laquelle est ainsi une équation à différences partielles du second ordre, et, cela étant, on a pour $d, e, f$ les expressions mentionnées, qui contiennent la fonction $\theta$, fonction qui n'est pas déterminée par les équations (2), [2].

L'équation [2], sous la forme abrégée, est

$$
x_{1} d_{2}+x_{2} d_{1}+a_{1} a_{2}=0,
$$

c'est-à-dire

$$
x_{1}[\lambda(\theta X+A)]_{2}+x_{2}[\lambda(\theta X+A)]_{1}+2 a_{1} a_{2}=0,
$$

ou, ce qui est la même chose,

$$
\lambda\left[x_{1}(\theta X+A)_{2}+x_{2}(\theta X+A)_{1}\right]+\lambda_{2} x_{1}(\theta X+A)+\lambda_{1} x_{2}(\theta X+A)+2 a_{1} a_{2}=0 .
$$

Les termes en $\theta$ sont

$$
\lambda\left[x_{1}\left(\theta X_{2}+\theta_{2} X\right)+x_{2}\left(\theta X_{1}+\theta_{1} X\right)\right]+\lambda_{2} \theta x_{1} X+\lambda_{1} \theta x_{2} X .
$$

qui s'évanouissent d'eux-mêmes; l'équation se réduit donc à

$$
\lambda\left(A_{2} x_{1}+A_{1} x_{2}\right)+\lambda_{2} A x_{1}+\lambda_{1} A x_{2}+2 a_{1} a_{2}=0,
$$

ou, en substituant la valeur de $a_{1} a_{2}$,

$$
\lambda\left(A_{2} x_{1}+A_{1} x_{2}\right)+\lambda_{2} A x_{1}+\lambda_{1} A x_{2}+2(\lambda X)_{1}(\lambda X)_{2}=0 ;
$$

on a

$$
(\lambda X)_{1}(\lambda X)_{2}=\left(\lambda X_{1}+\lambda_{1} X\right)\left(\lambda X_{2}+\lambda_{2} X\right),=\lambda_{2} X_{1} X_{2}+\lambda \lambda_{2} X X_{1}+\lambda \lambda_{1} X X_{2}+\lambda_{1} \lambda_{2} X^{2},
$$

et l'on trouve sans peine $A x_{1}=-a_{1} X, A x_{2}=-a_{2} X$, et de là

$$
\begin{aligned}
& A x_{1}=-(\lambda X)_{1} X,=-\lambda_{1} X^{2}-\lambda X X_{1}, \\
& A x_{2}=-(\lambda X)_{2} X,=-\lambda_{2} X^{2}-\lambda X X_{2} .
\end{aligned}
$$

Substituant ces valeurs, l'équation entière contiendra le facteur $\lambda$, et en l'écartant, elle devient

$$
A_{2} x_{1}+A_{1} x_{2}+\lambda_{2} X X_{1}+\lambda_{1} X X_{2}+2 \lambda X_{1} X_{2}=0 .
$$


Pour abréger encore la notation, au lieu de $x_{1}^{2},\left(=x_{1}{ }^{2}+y_{1}{ }^{2}+z_{1}^{2}\right)$, j'écris simplement 11, et ainsi dans les cas semblables: savoir, je me sers des abréviations

$$
\begin{aligned}
& 11=x_{1}{ }^{2}+y_{1}{ }^{2}+z_{1}{ }^{2}, \\
& 12=x_{1} x_{2}+y_{1} y_{2}+z_{1} z_{2}(=0),
\end{aligned}
$$

et je remarque que l'équation $12=0$, en prenant les dérivées par rapport à $p, q$ respectivement, donne $15+24=0,23+14=0$, équations qui servent pour éliminer des formules les expressions 15 et 23 . Si pour un moment nous dénotons ainsi par 124 le déterminant $\left|\begin{array}{lll}x_{1} & y_{1} & z_{1} \\ x_{2} & y_{2} & z_{2} \\ x_{4} & y_{4} & z_{4}\end{array}\right|$, alors, en multipliant par les déterminants analogues 123 et 125 respectivement, l'équation $124=0$ donne

$$
\left|\begin{array}{ccc}
11 & \cdot & 14 \\
\cdot & 22 & 24 \\
51 & 52 & 54
\end{array}\right|=0,\left|\begin{array}{ccc}
11 & \cdot & 14 \\
\cdot & 22 & 24 \\
31 & 32 & 34
\end{array}\right|=0
$$

dont chacune est une équation à trois termes entre les quantités $11,22, \ldots$

Nous avons

$$
\begin{aligned}
A & =y_{1}(\lambda Z)_{2}-y_{2}(\lambda Z)_{1}+z_{2}(\lambda Y)_{1}-z_{1}(\lambda Y)_{2}, \\
& =\lambda\left(y_{1} Z_{2}-y_{2} Z_{1}+z_{2} Y_{1}-z_{1} Y_{2}\right)+\lambda_{1}\left(z_{2} Y-y_{2} Z\right)+\lambda_{2}\left(y_{1} Z-z_{1} Y\right)
\end{aligned}
$$

or nous avons

$$
Y, Z=z_{1} x_{2}-z_{2} x_{1}, x_{1} y_{2}-x_{2} y_{1},
$$

et en formant de là les valeurs de $Y_{1}, Y_{2}, Z_{1}, Z_{2}$ on obtient sans peine

$$
\begin{gathered}
A=\lambda\left[x_{1}(15-24)+x_{2}(23-14)-x_{3} .22+2 x_{4} .12-x_{5} .11\right] \\
+\lambda_{1}\left(x_{2} .12-x_{1} .22\right)+\lambda_{2}\left(x_{1} \cdot 12-x_{2} .11\right),
\end{gathered}
$$

ou, ce qui est la même chose,

$$
A=\lambda\left[-2 x_{1} .24-2 x_{2} .14-x_{3} .22-x_{5} .11\right]-\lambda_{1} x_{1} .22-\lambda_{2} x_{2} .11 .
$$

Écrivons pour un moment

nous avons

$$
A=\lambda P+\lambda_{1} P^{\prime}+\lambda_{2} P^{\prime \prime} ;
$$

et de là

$$
\begin{aligned}
& A_{1}=P_{1} \lambda+\left(P+P_{1}^{\prime}\right) \lambda_{1}+P_{1}^{\prime \prime} \lambda_{2}+P^{\prime} \lambda_{3}+P^{\prime \prime} \lambda_{4}, \\
& A_{2}=P_{2} \lambda+\quad P_{2}^{\prime} \lambda_{1}+\left(P+P_{2}^{\prime \prime}\right) \lambda_{2}+P^{\prime} \lambda_{4}+P^{\prime \prime} \lambda_{5},
\end{aligned}
$$

$$
\begin{aligned}
A_{1} x_{2}+A_{2} x_{1}= & \lambda\left(P_{1} x_{2}+P_{2} x_{1}\right)+\lambda_{1}\left[\left(P+P_{1}^{\prime}\right) x_{2}+P_{2}^{\prime} x_{1}\right] \\
& +\lambda_{2}\left[P_{1}^{\prime \prime} x_{2}+\left(P+P_{2}^{\prime \prime}\right) x_{1}\right]+\lambda_{3} P^{\prime} x_{2} \\
& +\lambda_{4}\left(P^{\prime \prime} x_{2}+P^{\prime} x_{1}\right)+\lambda_{5} P^{\prime \prime} x_{1} .
\end{aligned}
$$


[Pp. 381-385.]

Les expressions de $P_{1}, P_{2}, \ldots$ contiennent les dérivées du troisième ordre

$$
\frac{d^{3} x}{d p^{3}}=x_{6}, \quad \frac{d^{3} x}{d p^{2} d q}=x_{7}, \quad \frac{d^{3} x}{d p d q^{2}}=x_{8}, \quad \frac{d^{3} x}{d q^{3}}=x_{9}, \ldots
$$

En formant les dérivées des équations $23+14=0,15+24=0$ par rapport à $p$ et $q$ respectivement, on obtient

On obtient alors

$$
\begin{aligned}
& 17+26+2.34=0 \\
& 18+27+35+44=0 \\
& 19+28+2.45=0
\end{aligned}
$$

$$
\begin{aligned}
P_{1}= & -2 x_{1}(44+17)+2 x_{2}(34+17) \\
& -4 x_{3} .24-2 x_{4} .14-2 x_{5} .13-x_{6} .22-x_{8} .11
\end{aligned}
$$

et de là la somme $P_{1} x_{2}$ est

$$
=-2.22(34+17)-4.23 .24-2.24 .14-2.25 .13-26.22-28.11 \text {, }
$$

ou, ce qui est la même chose,

$$
P_{1} x_{2}=-22.17-11.28+2.14 .24-2.25 .13 .
$$

On a de même

$$
\begin{aligned}
P_{2}= & -2 \cdot x_{1}(45+28)-2 \cdot x_{2}(44+18) \\
& -2 x_{3} .25-2 x_{4} \cdot 24-4 x_{5} .14-x_{7} \cdot 22-x_{9} .11,
\end{aligned}
$$

et de là la somme $P_{2} x_{1}$ est

$$
=-2.11(45+28)-2.13 .25-2.14 .24-4.15 .14-17.22-19.11 \text {, }
$$

ou, ce qui est la même chose,

on a donc

$$
P_{2} x_{1}=-22.17-11.28+2.14 .24-2.25 .13\left(=P_{1} x_{2}\right) ;
$$

$$
P_{1} x_{2}+P_{2} x_{1}=-2.22 .17-2.11 .28+4.14 .24-4.25 .13 .
$$

On obtient sans peine les autres sommes

$$
\begin{aligned}
& P^{\prime} x_{2}=0, \quad P^{\prime \prime} x_{2}+P^{\prime} x_{1}=-2.11 .22, \quad P^{\prime \prime} x_{1}=0, \\
& P x_{1}=-11.24-22.13, \quad P x_{2}=-11.25-22.14
\end{aligned}
$$

et l'on a ainsi

$$
\begin{aligned}
A_{1} x_{2}+A_{2} x_{1}= & \lambda(-2.11 .28-2.22 .17+4.14 .24-4.25 .13) \\
& +\lambda_{1}(-3.11 .25-2.22 .14-22.23) \\
& +\lambda_{2}(-2.11 .24-11.15-3.22 .13) \\
& +\lambda_{4}(-2.11 .22)
\end{aligned}
$$


L'équation en $\lambda$ est

$$
A_{1} x_{2}+A_{2} x_{1}+2 \lambda X_{1} X_{2}+\lambda_{1} X X_{2}+\lambda_{2} X X_{1}=0
$$

et l'on obtient sans peine

$$
\begin{aligned}
& X_{1} X_{2}=11.45+22.34+3.14 \cdot 24+25.13 \\
& X X_{2}=11.25+22.14 \\
& X X_{1}=11.24+22.13
\end{aligned}
$$

Donc enfin l'équation en $\lambda$ est

$\lambda[11(-28+45)+22(-17+34)+3.14 .24-25.13]-\lambda_{1} .11 .25-\lambda_{2} \cdot 22.13-\lambda_{4} \cdot 11 \cdot 22=0$.

Cette équation est vérifiée par la valeur $R=\frac{1}{V}\left(V=\sqrt{X^{2}+Y^{2}+Z^{2}}\right)$; en effet, en dénotant pour un moment le premier coefficient par $\Lambda$, l'équation à vérifier est

$$
\Lambda V^{2}+11.25 . X X_{1}+22.13 \cdot X X_{2}+\left(X^{2} \cdot X_{1} X_{2}+X^{2} \cdot X X_{4}-3 \cdot X X_{1} \cdot X X_{2}\right)=0 \text {, }
$$

c'est-à-dire

$$
\begin{aligned}
11.22 \Lambda & +11.25(22.13+11.24)+22.13(22.14+11.25) \\
& +11.22\left(11.45+22.34+3.14 .24+24.13+X X_{4}\right) \\
& -3(22.13+11.24)(22.14+11.25)=0,
\end{aligned}
$$

et l'on remarque qu'il n'y a ici que les termes $-2\left(11^{2} \cdot 24.25+22^{2} \cdot 13.24\right)$ qui ne contiennent pas le facteur 11.22.

Savoir, l'équation est de la forme

$$
11.22 \Omega-2\left(11^{2} \cdot 24.25+22^{2} .13 \cdot 14\right)=0 ;
$$

mais, des équations mentionnées $123 \cdot 124=0$ et $125 \cdot 124=0$, on obtient

$$
\begin{aligned}
& 22^{2} \cdot 13 \cdot 14=11.22(22.34+14.24), \\
& 11^{2} \cdot 24.25=11.22(11.45+14.24) .
\end{aligned}
$$

Donc l'équation entière contient le facteur 11.22 et, en l'écartant, elle devient

On a

$$
\Omega-2(11.45+22.34+2.14 \cdot 24)=0 \text {. }
$$

$$
\Omega=11 .(-28+2.45)+22(-17+2.34)-25.13+3.14 .24+X X_{4}
$$

l'équation est done

$$
-11.28-22.17-25.13-14.25+X X_{4}=0 ;
$$

et l'on vérifie sans peine que la valeur de $X X_{4}$ est actuellement

$$
X X_{4}=11.28+22.17+25.13+14.24 \text {. }
$$


Donc, en écrivant $\lambda=\frac{\rho}{V}$, l'équation en $\rho$ ne contiendra que les termes en $\rho_{1}, \rho_{2}, \rho_{4}$. En effet, l'équation devient

$$
-11.25 \frac{\rho_{1}}{V}-22.13 \frac{\rho_{2}}{V}-V^{2}\left(\frac{\rho_{4}}{V}-\frac{\rho_{1}}{V^{3}} X X_{2}-\frac{\rho_{2}}{V^{3}} X X_{1}\right),
$$

où, comme auparavant, $X X_{1}$ dénote $X X_{1}+Y Y_{1}+Z Z_{1}$, et de même $X X_{2}$ dénote $X X_{2}+Y Y_{2}+Z Z_{2}$. Nous avons déjà trouvé

$$
X X_{1}=22.13+11.24, \quad X X_{2}=22.14+11.25 ;
$$

l'équation devient ainsi

$$
11.22 \rho_{4}-14.22 \rho_{1}-24.11 \rho_{2}=0 .
$$

Savoir, cette équation est

$$
2 \frac{d^{2} \rho}{d p d q}-\frac{1}{E} \frac{d E}{d q} \frac{d \rho}{d p}-\frac{1}{G} \frac{d G}{d p} \frac{d \rho}{d q}=0
$$

Pour compléter la solution, il convient d'exprimer $A, B, C$ en termes de $\rho$. Nous avons

$$
A=\lambda\left(-2 x_{1} .24-2 x_{2} .14-x_{3} .22-x_{5} .11\right)-\lambda_{1} x_{1} .22-\lambda_{2} x_{2} .11 .
$$

Substituant la valeur $\lambda=\frac{\rho}{V}$, le coefficient de $\rho$ est

$$
\begin{gathered}
\frac{1}{V}\left(-2 x_{2} .24-2 x_{2} .14-x_{3} .22-x_{5} .11\right)+\frac{1}{V^{3}}\left(x_{1} .22 X X_{1}+x_{2} .11 . X X_{2}\right) \\
=\frac{1}{V^{3}}\left[11.22\left(-2 x_{1} \cdot 24-2 x_{2} .14-x_{3} \cdot 22-x_{5} .11\right)\right. \\
\left.+x_{1} \cdot 22(13.22+24.11)+x_{2} .11(14.22+25.11)\right]
\end{gathered}
$$

ou, ce qui est la même chose

$$
\frac{1}{V^{3}}\left[x_{1} .22(22.13+11.15)+x_{2} .11(11.25+22.23)-11.22\left(x_{3} .22+x_{5} .11\right)\right] .
$$

Le terme entre [ ] est fonction linéaire de $x_{3}, y_{3}, z_{3}, x_{5}, y_{5}, z_{5}$, et en réunissant les termes qui contiennent ces quantités respectivement, on le réduit sans peine à la forme

$$
X\left[11\left(X x_{5}+Y y_{5}+Z z_{5}\right)+22\left(X x_{3}+Y y_{3}+Z z_{3}\right)\right],
$$

ou, ce qui est la même chose

$$
X(11.125+22.123) \text {. }
$$

Nous avons donc

$$
A=-\frac{\rho X}{V^{3}}(11.125+22.123)-\frac{1}{V}\left(x_{1} \rho_{1} .22+x_{2} \rho_{2} .11\right) .
$$


Nous avons

$$
11=E, \quad 22=G, \quad V=\sqrt{E G} ;
$$

donc, en écrivant, pour abréger,

$$
-\frac{\rho}{E G \sqrt{E G}}(11.125+22.123)=\theta^{\prime},
$$

la valeur est

$$
A=\theta^{\prime} X-\frac{1}{\sqrt{E G}}\left(G \frac{d x}{d p} \frac{d \rho}{d p}+E \frac{d x}{d q} \frac{d \rho}{d q}\right)
$$

avec des expressions semblables pour $B$ et $C$. Dans les expressions $2 d=\frac{\rho}{\sqrt{E G}}(\theta X+A) \ldots$, la fonction $\theta^{\prime}$ se combine avec la fonction arbitraire $\theta$, de manière qu'il serait permis de remplacer $\theta+\theta^{\prime}$ par un seul symbole $\theta$, mais je retiens $\theta+\theta^{\prime}$.

Donc, enfin, les expressions de $\xi, \eta, \zeta$ deviennent

$$
\begin{aligned}
& \xi=x+\frac{\rho X}{\sqrt{E G}} r+\frac{1}{2}\left[\frac{\left(\theta+\theta^{\prime}\right) \rho X}{\sqrt{E G}}-\frac{\rho}{E G}\left(G \frac{d x}{d p} \frac{d \rho}{d p}+E \frac{d x}{d q} \frac{d \rho}{d q}\right)\right] r^{2}+\ldots \\
& \eta=y+\frac{\rho Y}{\sqrt{E} G} r+\frac{1}{2}\left[\frac{\left(\theta+\theta^{\prime}\right) \rho Y}{\sqrt{E G}}-\frac{\rho}{E G}\left(G \frac{d y}{d p} \frac{d \rho}{d p}+E \frac{d y}{d q} \frac{d \rho}{d q}\right)\right] r^{2}+\ldots \\
& \zeta=z+\frac{\rho Z}{\sqrt{E G}} r+\frac{1}{2}\left[\frac{\left(\theta+\theta^{\prime}\right) \rho Z}{\sqrt{E G}}-\frac{\rho}{E G}\left(G \frac{d z}{d p} \frac{d \rho}{d p}+E \frac{d z}{d q} \frac{d \rho}{d q}\right)\right] r^{2}+\ldots
\end{aligned}
$$

Je remarque que l'on satisfait à toutes les conditions en prenant $\rho=$ const. (ou, ce qui est la même chose, $\rho=1), \theta+\theta^{\prime}=0$ : cela donne

$$
\xi, \eta, \zeta=x+\frac{r X}{\sqrt{E G}}, \quad y=\frac{r Y}{\sqrt{E G}}, \quad z=\frac{r Z}{\sqrt{E G}} ;
$$

savoir, la famille est ici celle des surfaces parallèles à la surface donnée.

[Pp. 1800-1803.]

J'ai trouvé que l'équation différentielle du troisième ordre, sous la forme [ci-dessus trouvée], contient le facteur étranger $X^{2}+Y^{2}+Z^{2}$, et que l'équation débarrassée de ce facteur devient beancoup plus simple. La réduction et aussi la nouvelle méthode dont je me suis servi pour obtenir l'équation réduite sont toutes les deux assez pénibles; mais cette nouvelle méthode a l'avantage d'établir un résultat intermédiaire qui a quelque valeur. J'ai changé un peu la notation; aussi je commence en l'expliquant.

Je prends $U=0$ l'équation d'une surface; $X, Y, Z$ les coefficients différentiels du premier ordre; $a, b, c, f, g, h$ les coefficients du second ordre;

$$
(A, B, C, F, G, H \gamma d x, d y, d z)^{2}=0
$$


l'équation différentielle des courbes de courbure; savoir:

et

$$
\begin{aligned}
& A=2(h Z-g Y), \\
& B=2(f X-h Z), \\
& C=2(g Y-f X), \\
& F=h Y-g Z-(b-c) X, \\
& G=f Z-h X-(c-a) Y, \\
& H=g X-f Y-(a-b) Z
\end{aligned}
$$

$[(A),(B),(C),(F),(G),(H)](d x, d y, d z)^{2}$

savoir :

$$
=(A, B, C, F, G, H \gamma Y d z-Z d y, Z d x-X d z, X d y-Y d x)^{2} ;
$$

$$
\begin{aligned}
& (A)=B Z^{2}+C Y^{2}-2 F Y Z, \\
& (B)=C X^{2}+A Z^{2}-2 G Z X, \\
& (C)=A Y^{2}+B X^{2}-2 H X Y, \\
& (F)=-a Y Z-f X^{2}+g X Y+h X Z, \\
& (G)=-b Z X+f Y X-g Y^{2}+h Y Z, \\
& (H)=-c X Y+f Z X+g Z Y-h Z^{2},
\end{aligned}
$$

ce qui explique la signification des symboles $(A),(B), \ldots$; aussi

$$
V^{2}=X^{2}+Y^{2}+Z^{2},
$$

et

$$
(\bar{a}, \bar{b}, \bar{c}, \bar{f}, \bar{g}, \bar{h})=\left(b c-f^{2}, c a-g^{2}, a b-h^{2}, g h-a f, h f-b g, f g-c h\right) .
$$

Cela étant, à chaque point $P$ de la surface $U=0$, je prends sur la normale une distance infinitésimale $P P^{\prime}=\rho$, où $\rho$ est une fonction quelconque des coordonnées $x, y, z$ du point $P$; on obtient ainsi une surface, lieu des points $P^{\prime}$, laquelle se nomme la surface voisine, et les points $P$ et $P^{\prime}$ sont des points correspondants sur les deux surfaces.

Pour que les deux surfaces forment partie d'un système orthogonal, je trouve que la distance $\rho$, considérée comme fonction des coordonnées $(x, y, z)$, doit satisfaire à cette équation différentielle du second ordre

$$
[(A),(B),(C),(F),(G),(H)]\left(d_{x}, d_{y}, d_{z}\right)^{2} \rho=0 .
$$

Or, si la surface donnée $U=0$ et la surface voisine sont des surfaces consécutives d'une famille $r-f(x, y, z)=0$; savoir, si l'équation de la surface donnée est $r-f(x, y, z)=0$, et celle de la surface voisine $r+d r-f(x, y, z)=0$, on a

$$
\rho=\frac{d r}{V}, \quad V=\sqrt{X^{2}+Y^{2}+Z^{2}},
$$

où $d r$ est une constante; l'équation devient ainsi

$$
[(A), \ldots]\left(d_{x}, d_{y}, d_{z}\right)^{2} \frac{1}{V}=0,
$$

C. VIII. 
où, à présent, $X, Y, Z, a, b, c, f, g, h$ dénotent les coefficients différentiels de $f(x, y, z)$, ou (ce qui est la même chose) du paramètre $r$, considéré comme fonction des coordonnées $x, y, z$. Cette équation est donc une équation du troisième ordre, à laquelle doit satisfaire la fonction $\rho$; multipliée par $V^{5}$, elle est en effet l'équation [dont il s'agit], laquelle, comme j'ai déjà dit, contient le facteur $V^{2}$ : donc pour écarter le dénominateur, il suffira de multiplier par $V^{3}$.

J'écris $\delta=X d_{x}+Y d_{y}+Z d_{z}$,

et de là

$$
\delta X, \delta Y, \delta Z=a X+h Y+g Z, \quad h X+b Y+f Z, \quad g X+f Y+c Z,
$$

respectivement. On trouve

$$
\begin{array}{r}
d_{x}^{2} \frac{1}{V}=-\frac{1}{V^{3}}\left(a^{2}+h^{2}+g^{2}+\delta a\right)+\frac{3}{V^{5}}(\delta X)^{2}, \\
d_{y} d_{x} \frac{1}{V}=-\frac{1}{V^{3}}(g h+b f+c f+\delta f)+\frac{3}{V^{5}} \delta Y \delta Z,
\end{array}
$$

ou, en écrivant $\omega=a+b+c, \bar{\omega}=\bar{a}+\bar{b}+\bar{c}$, ces valeurs deviennent

$$
\begin{aligned}
& d_{x}{ }^{2} \frac{1}{V}=-\frac{1}{V^{3}}(a \omega-\bar{\omega}+\bar{a}+\delta a)+\frac{3}{V^{5}}(\delta X)^{2}, \\
& d_{y} d_{x} \frac{1}{V}=-\frac{1}{V^{3}}(f \omega+\bar{f}+\delta f)+\frac{3}{V^{5}} \delta Y \delta Z .
\end{aligned}
$$

et, en substituant ces valeurs, les termes en $\omega, \bar{\omega}$ disparaissent d'eux-mêmes, et l'équation, multipliée seulement par $-V^{3}$, se réduit à

$$
[(A), \ldots](\bar{a}, \ldots)+[(A), \ldots](\delta a, \ldots)-\frac{3}{V^{2}}[(A), \ldots](\delta X, \delta Y, \delta Z)^{2}=0
$$

où le premier terme est

$$
(A) \bar{a}+(B) \bar{b}+(C) \bar{c}+2\left(F^{\prime}\right) \bar{f}+2(G) \bar{g}+2(H) \bar{h},
$$

et de même pour le second terme.

Or je trouve que l'on a identiquement

$$
[(A), \ldots](\delta X, \delta Y, \delta Z)^{2}=-V^{2}(A, \ldots \gamma \delta X, \delta Y, \delta Z)^{2},
$$

de manière que l'équation est

$$
[(A), \ldots](\bar{a}, \ldots)+[(A), \ldots](\delta a, \ldots)+3(A, \ldots)(\delta X, \delta Y, \delta Z)^{2}=0
$$

et, de plus, que l'on a identiquement

$$
[(A), \ldots](\bar{a}, \ldots)=-(A, \ldots)(\delta X, \delta Y, \delta Z)^{2}=2\left|\begin{array}{rrr}
\delta X, & \delta Y, & \delta Z \\
X, & Y, & Z \\
\bar{\delta} X, & \bar{\delta} Y, & \bar{\delta} Z
\end{array}\right|
$$


où $\bar{\delta} X, \bar{\delta} Y, \bar{\delta} Z$ dénotent respectivement $\bar{a} X+\bar{h} Y+\bar{g} Z, \bar{h} X+\bar{b} Y+\bar{f} Z, \bar{g} X+\bar{f} Y+\bar{c} Z$;

l'équation se réduit donc à

$$
[(A), \ldots](\delta a, \ldots)+\Omega=0,
$$

où $\Omega$ peut être exprimé à volonté sous l'une quelconque des trois formes

$$
\begin{aligned}
& =+2[(A), \ldots](\bar{a}, \ldots), \\
& =+2(A, \ldots \gamma \delta X, \delta Y, \delta Z)^{2}, \\
& =-4\left|\begin{array}{rrr}
\delta X, & \delta Y, & \delta Z \\
X, & Y, & Z \\
\bar{\delta} X, & \bar{\delta} Y, & \bar{\delta} Z
\end{array}\right| .
\end{aligned}
$$

Prenant la première forme, l'équation est

$$
[(A), \ldots](\delta a, \ldots)-2[(A), \ldots](\bar{a}, \ldots)=0,
$$

ou, ce qui est la même chose,

$$
\text { (A) } \begin{aligned}
\delta a+(B) \delta b+(C) \delta c+2\left(F^{\prime}\right) \delta f+2(G) \delta g+2(H) \delta h \\
-2[(A) \bar{a}+(B) \bar{b}+(C) \bar{c}+2(F) \bar{f}+2(G) \bar{g}+2(H) \bar{h}]=0,
\end{aligned}
$$

où les coefficients sont des fonctions données de $X, Y, Z, a, b, c, f, g, h$, les coefficients différentiels de $r$ du premier et du second ordre, et $\delta$ dénote $X d_{x}+Y d_{y}+Z d_{z}$. 